\title{
Energy State Amplification in an Energy Harvesting Communication System
}

\author{
Omur Ozel Sennur Ulukus \\ Department of Electrical and Computer Engineering \\ University of Maryland College Park, MD 20742 \\ omur@umd.edu \\ ulukus@umd.edu
}

\begin{abstract}
In energy harvesting communication systems, the energy required for message transmission is maintained by an exogenous energy arrival process independent of the message. This links the problem of communication with an energy harvesting transmitter to the problem of communication over statedependent channels. In particular, if the transmitter has no battery, the available energy can be viewed as a state and the resulting channel is a state-dependent channel with causal state information at the transmitter only. In general, information transmission blurs the state information that the receiver can get from the received signal. In this paper, we explore the trade-off between the information rate $R$ and the entropy reduction of the energy arrival process $\Delta$ at the receiver side over an AWGN channel with an energy harvesting transmitter. If the transmitter has no battery, the trade-off points are achieved by Shannon strategies and we show that the optimal input distributions are discrete. Next, we consider the state amplification problem for an energy harvesting transmitter with an unlimited battery. We show that the optimal trade-off region in this extreme case is expressed explicitly in a simple form and its boundary is achieved by a combination of best-effort-transmit and random binning schemes with an i.i.d. Gaussian codebook of average power equal to the average recharge rate. Finally, we propose an uncoded state amplification scheme that splits the energy between message transmission and entropy reduction and study its performance in a numerical example.
\end{abstract}

\section{INTRODUCTION}

Energy harvesting is a promising technology for many wireless networking applications as it brings self-sustainability and practically unlimited lifetime. In applications where transmitter has an energy harvesting capability, energy needed to send messages is replenished stochastically throughout the communication session. From an information-theoretic point of view, the stochasticity of the energy at the transmitter side connects this setting to state-dependent channels [1], [2]: energy can be viewed as a state of the channel that is perfectly known to the transmitter but unknown to the receiver. In many applications, e.g., energy harvesting sensors, the receiver may aim at extracting energy state information from the received signal as well as decoding the message. In this paper, we explore the interaction of these two objectives.

As shown in Fig. 1, we consider an energy harvesting transmitter with an unlimited battery or no battery sending messages over an additive white Gaussian noise (AWGN) channel. An exogenous energy source supplies $E_{i}$ amount

This work was supported by NSF Grants CCF 07-29127, CNS 09-64632, CCF 09-64645, CCF 10-18185 and CNS 11-47811.
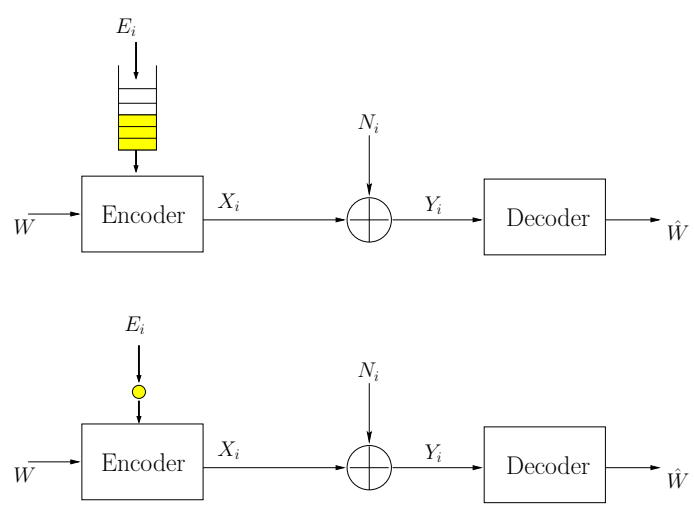

Fig. 1. The AWGN channel with an energy harvesting transmitter that has an unlimited battery (on the top) and no battery (on the bottom).

of energy at the beginning of the $i$ th channel use and upon observing the arrived energy, the transmitter sends a code symbol whose energy is constrained to the currently available energy. The channel input and output are related as

$$
Y_{i}=X_{i}+N_{i}, \quad i=1, \ldots, n
$$

where $X_{i}$ is the channel input, $Y_{i}$ is the channel output, and $N_{i}$ is the i.i.d. zero-mean unit-variance Gaussian noise, in the $i$ th channel use. $E_{1}, \ldots, E_{n}$ is the i.i.d. energy arrival sequence which is independent of the message. The code symbol energy at the $i$ th channel use is constrained according to the exogenous energy arrival and the availability of a battery (energy buffer) at the transmitter. In particular, if there is no battery at the transmitter, the transmitter observes $E_{i}$ and generates a channel input $X_{i}$ that satisfies $X_{i}^{2} \leq E_{i}$, i.e., the code symbol is amplitude constrained to (the square root of) the observed energy. In [1], [2], we addressed this system and found the capacity achieving scheme by solving for the maximum mutual information between the input and the output of an extended input channel. More specifically, we proved in [1], [2] that the code symbols need to be chosen from a finite set in the capacity achieving scheme analogous to [3]. In the other extreme, if the transmitter has an unlimited battery, some portion of the arriving energy can be stored in the battery and the code symbol energy at the $i$ th channel use is constrained to the energy in the battery at the beginning of the $i$ th channel use. We studied this problem in [4], [5] and found that the capacity of this system is equal to the capacity 
with average power constrained to the average recharge rate and it is achieved by save-and-transmit or best-effort-transmit schemes using a Gaussian codebook.

In [1], [2], [4], [5], the sole purpose of the transmitter is to convey the message which is independent of the energy arrival process. However, the transmitter may help the receiver get some information about the energy arrival process at the transmitter. In this paper, we analyze the interaction between the message transmission rate and the receiver's information about the energy arrival process at the transmitter. In particular, there is a trade-off between these two objectives in view of the connection of this setting with state-dependent channels with causal state information at the transmitter. This tradeoff has been well studied for state-dependent channels with causal or noncausal state information at the transmitter [6] [10] where the information the receiver can learn about the state is measured by different metrics.

In this paper, we use entropy reduction metric used in [7] and characterize the fundamental trade-off between the entropy reduction $\Delta$ of transmitter's energy arrivals at the receiver and the message transmission rate $R$ in an energy harvesting communication system with causal energy state information at the transmitter only. When the transmitter has no battery, we find the optimal $(R, \Delta)$ trade-off points using Shannon strategies and prove that the optimal input distributions are discrete. When the transmitter has an unlimited battery, we show that the optimal trade-off region has a simple form. Specifically, no information about the energy arrival process at the transmitter can be obtained at the receiver when the system is operated at the highest message rate. Finally, we propose an uncoded state amplification scheme that splits the energy between message transmission and entropy reduction.

\section{State Amplification with a Batteryless Energy HARVESTING TRANSMITTER}

In this section, we consider a batteryless energy harvesting transmitter as shown in the bottom figure of Fig. 1. For brevity, we consider a binary energy arrival process with alphabet $\mathcal{E}=$ $\left\{e_{1}, e_{2}\right\}$ and probabilities $P\left(E_{i}=e_{1}\right)=p_{e_{1}}$ and $P\left(E_{i}=\right.$ $\left.e_{2}\right)=p_{e_{2}}$ for all $i$.

As the energy at each channel use varies as an i.i.d. process and is independent of the message $w \in \mathcal{W}$, the resulting channel is a state-dependent channel with causal state information at the transmitter [1], [2], [11]. The transmitter helps the receiver estimate the energy arrived at the transmitter's side while sending a message $w \in \mathcal{W}$ at the same time where $|\mathcal{W}|=2^{n R}$. The receiver forms a list $L_{n}\left(Y^{n}\right) \subset \mathcal{E}^{n}$ of possible energy arrival sequences upon receiving the sequence $Y^{n}$. Before receiving $Y^{n}$, the size of the list is $2^{n H(E)}$, the size of the typical set of energy arrival sequences. Upon receiving $Y^{n}$, the list size is dropped to $\left|L_{n}\left(Y^{n}\right)\right|$. Hence, the energy arrival sequence uncertainty reduction rate is

$$
\Delta=\frac{1}{n}\left(H\left(E^{n}\right)-\log _{2}\left|L_{n}\left(Y^{n}\right)\right|\right)
$$

A $\left(2^{n R}, 2^{n \Delta}, n\right)$ code is composed of an encoder map $X^{n}$ :
$\mathcal{W} \times \mathcal{E}^{n} \rightarrow \mathcal{R}^{n}$ where $X_{i}=\mathcal{W} \times \mathcal{E}^{i} \rightarrow \mathcal{R}, i=1, \ldots, n$ since only causal information of energy arrivals is available. In particular, $\left|X_{i}\left(w, E_{i}\right)\right| \leq \sqrt{E_{i}}$ for all $w \in \mathcal{W}$ and $E_{i} \in \mathcal{E}$. The receiver performs two decoding operations after receiving the sequence $Y^{n}$ : decoding the message $w \in \mathcal{W}$ and list decoding the energy arrival sequence $\left\{E_{i}\right\}_{i=1}^{n}$. A rate-entropy reduction pair $(R, \Delta)$ is achievable if there exists a sequence of $\left(2^{n R}, 2^{n \Delta}, n\right)$ codes with probabilities of message and list decoding errors converging to zero as the block length is increased. The optimal trade-off region $\mathcal{R}$ is the closure of all achievable $(R, \Delta)$ pairs.

We first note that $\mathcal{R}$ is a convex region [7]. In view of [7, Theorem 2] and replacing the auxiliary variable $U$ with Shannon strategy $\left(T_{1}, T_{2}\right)$ [11] where $T_{i}$ is the channel input when energy $E_{i}$ is observed, the region $\mathcal{R}$ is characterized as

$$
\begin{aligned}
R & \leq I\left(T_{1}, T_{2} ; Y\right) \\
\Delta & \leq H(E) \\
R+\Delta & \leq I(X, E ; Y)
\end{aligned}
$$

for some $\left(T_{1}, T_{2}\right)$ with amplitude constraints $\left|T_{1}\right| \leq \sqrt{E_{1}}$, $\left|T_{2}\right| \leq \sqrt{E_{2}}$ and

$$
p\left(y \mid t_{1}, t_{2}\right)=p_{e_{1}} \phi\left(y-t_{1}\right)+p_{e_{2}} \phi\left(y-t_{2}\right)
$$

where $\phi(y)=\frac{1}{\sqrt{2 \pi}} e^{-\frac{y^{2}}{2}}$. We denote the interval $\left[-\sqrt{e_{i}}, \sqrt{e_{i}}\right]$ as $\mathcal{T}_{i}, i=1,2$. The received signal has pdf $p(y)$

$p(y)=\int_{\mathcal{T}_{1}} \int_{\mathcal{T}_{2}}\left(p_{e_{1}} \phi\left(y-t_{1}\right)+p_{e_{2}} \phi\left(y-t_{2}\right)\right) d F_{T_{1}, T_{2}}\left(t_{1}, t_{2}\right)$

If the goal of the encoder is only to transmit messages and not to assist the receiver to list decode the energy arrival sequence, the maximum achievable rate $C_{0}$ is found as [1], [2]

$$
C_{0}=\max _{F_{T_{1}, T_{2}} \in \Omega} I\left(T_{1}, T_{2} ; Y\right)
$$

where the set of feasible distributions is

$$
\Omega=\left\{F: \int_{\mathcal{T}_{1}} \int_{\mathcal{T}_{2}} d F\left(t_{1}, t_{2}\right)=1\right\}
$$

On the other extreme, if the goal of the encoder is only to amplify the arrived energy, optimal reduction in the entropy is

$$
\Delta^{*}=\min \left\{H(E), \max _{F_{T_{1}, T_{2}} \in \Omega} I(X, E ; Y)\right\}
$$

Note that $I(X, E ; Y)=h(Y)-\frac{1}{2} \log _{2}(2 \pi e)$, that is, $h(Y \mid X, E)$ is equal to the entropy of the Gaussian noise.

\section{DisCRETENESS OF THE OPTIMAL INPUT DISTRIBUTIONS}

As $\mathcal{R}$ is a convex region and due to its characterization in (3)-(5), one can show after algebraic rearrangements that the boundary of $\mathcal{R}$ is obtained by solving the following optimization problems for all $\mu \geq 0$ :

$$
\max _{F_{T_{1}, T_{2}} \in \Omega} \mu I\left(T_{1}, T_{2} ; Y\right)+h(Y)
$$

A main conclusion we draw in this paper is that the optimal input distributions for all $\mu \geq 0$ are discrete: 
Theorem 1 For all $\mu \geq 0$, the optimal input distribution, i.e., the solution of (10), has a finite support set.

The proof of Theorem 1 follows from steps that have been followed in [3], [12], [13] and the finiteness claim is proved using a contradiction argument. The problem in (10) is a functional convex optimization problem. As a first step, we show that the space of feasible distributions $\Omega$ is a convex and compact set and the objective function in (10) is concave in the input distribution in the weak topology. Next, we obtain an optimality condition in terms of the mutual information density, the entropy density and the support set of the optimal input distribution. Then, we prove that the mutual information density and the entropy density have analytic extensions over $\mathbb{C}^{2}$. Finally, by invoking the identity theorem for analytic functions, we show that the optimality condition causes a contradiction when the support set is assumed infinite. In particular, the mutual information density and entropy density are given, respectively, as

$$
\begin{aligned}
& i\left(t_{1}, t_{2} ; F\right)=\int_{-\infty}^{\infty} \log _{2}\left(\frac{f\left(y \mid t_{1}, t_{2}\right)}{f(y ; F)}\right) f\left(y \mid t_{1}, t_{2}\right) d y \\
& h\left(t_{1}, t_{2} ; F\right)=-\int_{-\infty}^{\infty} \log _{2}(f(y ; F)) f\left(y \mid t_{1}, t_{2}\right) d y
\end{aligned}
$$

where $i\left(t_{1}, t_{2} ; F\right)$ and $h\left(t_{1}, t_{2} ; F\right)$ are continuous functions in $\mathbb{R}^{2}$ and they both have analytic extensions over $\mathbb{C}^{2}$. Moreover, $\Omega$ is convex and compact; $I\left(T_{1}, T_{2} ; Y\right)$ and $h(Y)$ are both concave and weakly differentiable functionals of $F$. Therefore, we have the following theorem:

Theorem 2 For the optimal input distribution $F_{T_{1}, T_{2}}^{*}$, we have

$$
\begin{aligned}
\mu i\left(t_{1}, t_{2} ; F^{*}\right)+h\left(t_{1}, t_{2} ; F^{*}\right) \leq & \mu I\left(F^{*}\right)+h\left(F^{*}\right), \\
& \forall\left(t_{1}, t_{2}\right) \in \mathcal{T}_{1} \times \mathcal{T}_{2} \\
\mu i\left(t_{1}, t_{2} ; F^{*}\right)+h\left(t_{1}, t_{2} ; F^{*}\right)= & \mu I\left(F^{*}\right)+h\left(F^{*}\right), \\
& \forall\left(t_{1}, t_{2}\right) \in \mathcal{S}_{F^{*}}
\end{aligned}
$$

where $\mathcal{S}_{F^{*}}$ is the support set of $F^{*}$.

The proof of the finiteness of $\mathcal{S}_{F^{*}}$ is by the following contradiction argument. Assume that $\mathcal{S}_{F^{*}}$ is infinite. By compactness of $\mathcal{T}_{1} \times \mathcal{T}_{2}, \mathcal{S}_{F^{*}}$ has an accumulation point and by the identity theorem for multi-dimensional complex numbers, we have

$$
\mu i\left(z_{1}, z_{2}\right)+h\left(z_{1}, z_{2}\right)=\mu I\left(F^{*}\right)+h\left(F^{*}\right), \quad \forall\left(z_{1}, z_{2}\right) \in \mathbb{C}^{2}
$$

In particular, $\mu i\left(t_{1}, t_{2}\right)+h\left(t_{1}, t_{2}\right)=\mu I\left(F^{*}\right)+h\left(F^{*}\right)$ for all $\left(t_{1}, t_{2}\right) \in \mathbb{R}^{2}$. For $t_{1}=t_{2}=t$, we obtain for all $t \in \mathbb{R}$

$$
\begin{aligned}
\int_{-\infty}^{\infty} \phi & (y-t) \log _{2}(p(y ; F)) d y \\
& =-\frac{1}{\mu+1}\left(\mu I\left(F^{*}\right)-\frac{\mu}{2} \log _{2}(2 \pi e)-h\left(F^{*}\right)\right)
\end{aligned}
$$

For all $\mu \geq 0$, the right hand side of (15) is a constant and in view of [13, Corollary 9], $\log _{2}(p(y ; F))$ is constant for all $y \in \mathbb{R}$, which is impossible as $p(y ; F)$ is a probability density function, yielding a contradiction, and consequently, proving the finiteness claim in Theorem 1 .

\section{State Amplification With an EnERgy HARVESTING TRANSMITTER OF UNLIMITED BATTERY}

In this section, we consider the state amplification problem with an energy harvesting transmitter that has an unlimited battery [4], [5] as shown in the top figure of Fig. 1. At each channel use, the energy arrival replenishes, while the code symbol energy reduces, the battery energy. Hence, the code symbol at the beginning of a channel use is constrained by the current energy level in the battery:

$$
\sum_{i=1}^{k} X_{i}^{2} \leq \sum_{i=1}^{k} E_{i}, \quad k=1, \ldots, n
$$

We assume that the transmitter has only causal information; however, it will be clear that the trade-off region is invariant under causal or noncausal information. At the $i$ th channel use, the transmitter has the observations $E_{1}, \ldots, E_{i}$ and determines the code symbol accordingly. The state amplification problem in this setting is to characterize the achievable information rate $R$ and entropy reduction $\Delta$ of the energy arrival sequence at the receiver side under the code symbol constraints in (16).

We found in [4], [5] that the maximum rate of information achievable under the input constraints in (16) and causal or noncausal information of the energy arrival information is

$$
C_{\infty}=\frac{1}{2} \log _{2}\left(1+\mathbb{E}\left[E_{i}\right]\right)
$$

In addition, the entropy reduction is bounded by the entropy of the energy arrival process as $\Delta \leq H(E)$. It remains to determine the bound on $R+\Delta$. We can bound $R+\Delta$ as

$$
\begin{aligned}
n(R+\Delta) & \leq I\left(W ; Y^{n}\right)+I\left(E^{n} ; Y^{n}\right)+n \epsilon_{n} \\
& \leq I\left(W ; Y^{n} \mid E^{n}\right)+I\left(E^{n} ; Y^{n}\right)+n \epsilon_{n} \\
& \leq I\left(W, E^{n} ; Y^{n}\right)+n \epsilon_{n} \\
& \leq I\left(X^{n}, E^{n} ; Y^{n}\right)+n \epsilon_{n} \\
& \leq \sum_{i=1}^{n} I\left(X_{i}, E_{i} ; Y_{i}\right)+\epsilon_{n}
\end{aligned}
$$

where (19) is due to the independence of the message $W$ and the energy arrival $E$ and conditioning reduces entropy, (21) is due to the data processing inequality and the fact that $X_{i}$ is a function of $W$ and $E_{1}, \ldots, E_{i}$, and (22) is due to the memoryless property of the AWGN channel. We note that $I\left(X_{i}, E_{i} ; Y_{i}\right)=h\left(Y_{i}\right)-\frac{1}{2} \log _{2}(2 \pi e)$. Hence, we get:

$$
\begin{aligned}
R+\Delta & \leq \frac{1}{n} \sum_{i=1}^{n} h\left(Y_{i}\right)-\frac{1}{2} \log _{2}(2 \pi e) \\
& \leq \frac{1}{n} \sum_{i=1}^{n} \frac{1}{2} \log _{2}\left(2 \pi e \mathbb{E}\left[Y_{i}^{2}\right]\right)-\frac{1}{2} \log _{2}(2 \pi e) \\
& \leq \frac{1}{2} \log _{2}\left(1+\mathbb{E}\left[E_{i}\right]\right)
\end{aligned}
$$

where (24) is due to the fact that Gaussian distribution maximizes entropy, and (25) is due to the concavity of $\log _{2}(1+x)$ and since $\sum_{i=1}^{n} \mathbb{E}\left[Y_{i}^{2}\right] \leq n \mathbb{E}\left[E_{i}\right]+n$, which follows from the constraints in (16). 
On the other hand, the bound in (25) is achievable by a combination ${ }^{1}$ of the best-effort-transmit scheme in [4], [5] with the random binning in [7]. In particular, we consider a blockby-block encoding scheme of $B$ blocks; each block is of $n$ channel uses. We consider a single i.i.d. Gaussian codebook with average power $\mathbb{E}\left[E_{i}\right]-\epsilon$ composed of $2^{n \frac{1}{2} \log _{2}\left(1+\mathbb{E}\left[E_{i}\right]-\epsilon\right)}$ codewords with block length $n$. In each block, we allocate $0 \leq R \leq \frac{1}{2} \log _{2}\left(1+\mathbb{E}\left[E_{i}\right]-\epsilon\right)$ bits for the message transmission and remaining $\Gamma=\frac{1}{2} \log _{2}\left(1+\mathbb{E}\left[E_{i}\right]-\epsilon\right)-R$ bits for state amplification. Hence, we have $2^{n R}$ bins each composed of $2^{n \Gamma}$ sequences, i.e., we divide the index interval $[1$ : $\left.2^{n \frac{1}{2} \log _{2}\left(1+\mathbb{E}\left[E_{i}\right]-\epsilon\right)}\right]$ into $2^{n R}$ intervals $\left[w 2^{n \Gamma}:(w+1) 2^{n \Gamma}\right]$, $w=1, \ldots, 2^{n R}-1$ where $w$ is a message index. In the first block, an arbitrary codeword independent of the energy arrival sequence is sent. The transmitter observes the energy arrival sequence $E_{1}, \ldots, E_{n}$, maps it to one of $2^{n \Gamma}$ indices independent of the message $w$. Then, according to the chosen message index $w$, the codeword to be sent is determined. The transmitter uses the best-effort-transmit scheme: if the energy of the code symbol $X_{i}$ in the $i$ th channel use is higher than the energy in the battery $E_{b_{i}}$ (i.e., $X_{i}^{2}>E_{b_{i}}$ ), then a zero symbol is put; otherwise, the code symbol $X_{i}$ is sent as it is. The codeword $X_{1}, \ldots, X_{n}$ is sent with only finitely many mismatches as $X_{i}^{2}>E_{b_{i}}$ occurs only in finitely many channel uses and this causes no error in the decoding of the sent codeword [4], [5]. As $X_{1}, \ldots, X_{n}$ is decoded at the receiver side with vanishing probability of error, the receiver recovers the message index $w$ and the bin index for the observed energy arrival sequence as the block length $n$ gets larger. If we allow $B$ increase and $\epsilon \rightarrow 0$, we have $R+\Delta \leq \frac{1}{2} \log _{2}\left(1+\mathbb{E}\left[E_{i}\right]\right)$.

Theorem 3 In an energy harvesting transmitter with an unlimited battery, the optimal $(R, \Delta)$ region is:

$$
\begin{aligned}
\Delta & \leq H(E) \\
R+\Delta & \leq \frac{1}{2} \log _{2}(1+\mathbb{E}[E])
\end{aligned}
$$

We observe from Theorem 3 that the optimal trade-off region in the unlimited battery case is expressed explicitly in a simple form and the maximum entropy reduction $\Delta^{*}$ is

$$
\Delta^{*}=\min \left\{H(E), \frac{1}{2} \log _{2}(1+\mathbb{E}[E])\right\}
$$

We also observe that in the unlimited battery case the entropy reduction is zero when the transmitter operates at the information transmission capacity $\frac{1}{2} \log _{2}(1+\mathbb{E}[E])$. In this case, the received sequence $Y^{n}$ is almost independent of the energy arrival profile $E^{n}$ even though the message transmission is enabled by the energy $E^{n}$. Therefore, the unlimited sized energy queue acts as an information hider [14] and the receiver can get no information about the energy arrival sequence if the message transmission is performed at the capacity. Finally, the $(R, \Delta)$ region in Theorem 3 remains unchanged if the transmitter had noncausal information of the energy arrivals.

\footnotetext{
${ }^{1}$ We might also achieve it using the save-and-transmit scheme instead of the best-effort-transmit scheme in [4], [5].
}

\section{A Simple Uncoded State Amplification Scheme}

In this section, we propose a suboptimal uncoded state amplification scheme based on the power splitting scheme in [6]. Pure state amplification in the energy harvesting communication context is just putting a code symbol of energy equal to the observed energy. The transmitter puts the channel symbol $\sqrt{e_{1}}$ when $e_{1}$ is observed and $-\sqrt{e_{2}}$ when $e_{2}$ is observed. This scheme corresponds to the deterministic auxiliary selection at $\left(T_{1}, T_{2}\right)=\left(\sqrt{e_{1}},-\sqrt{e_{2}}\right)$. We denote the entropy reduction in the uncoded transmission as $\Delta_{u c}$.

$$
\Delta_{u c}=h(Y)-\frac{1}{2} \log _{2}(2 \pi e)
$$

where $p(y)=p_{e_{1}} \phi\left(y-\sqrt{e_{1}}\right)+p_{e_{2}} \phi\left(y+\sqrt{e_{2}}\right)$. Note that the message transmission rate in this uncoded state amplification scheme is zero. In addition, all energy is utilized immediately after it is observed and hence the existence of a battery does not affect the performance.

Next, we propose an energy splitting scheme for simultaneous information transmission and entropy reduction. Upon observing energy $e_{i}, \alpha e_{i}$ is allocated for state amplification and $(1-\alpha) e_{i}$ is allocated for message transmission where $0 \leq \alpha \leq 1$. The transmitter puts $\alpha e_{1}$ when $e_{1}$ is observed and $-\alpha e_{2}$ when $e_{2}$ is observed with the goal of entropy reduction. The remaining energy is allocated for message transmission. When the transmitter has no battery, the channel is

$$
Y_{i}=X_{i}+\alpha E_{i}+N_{i}
$$

where $\left|X_{i}\right| \leq \sqrt{(1-\alpha) e_{1}}$ if $e_{1}$ is observed and $\left|X_{i}\right| \leq$ $\sqrt{(1-\alpha) e_{2}}$ if $e_{2}$ is observed. Hence, we find the optimal input distribution of the following extended input channel:

$$
p\left(y \mid \bar{t}_{1}, \bar{t}_{2}\right)=p_{e_{1}} \phi\left(y-\bar{t}_{1}-\sqrt{\alpha e_{1}}\right)+p_{e_{2}} \phi\left(y-\bar{t}_{2}+\sqrt{\alpha e_{2}}\right)
$$

where $\left|\bar{t}_{i}\right| \leq \sqrt{(1-\alpha) e_{i}}$. We note that the capacity achieving scheme for this extended input alphabet channel is also discrete which can be proved using similar steps and the contradiction argument. For given $\alpha$, the message transmission rate $R$ is the capacity of the channel in (31) and the resulting $\Delta$ is the maximum entropy reduction subject to the message transmission rate $R$. These values are found by evaluating the region for the original channel in (3)-(5) at $\left(t_{1 i}, t_{2 i}\right)=\left(\bar{t}_{1 i}^{*}, \bar{t}_{2 i}^{*}\right)+\left(\alpha \sqrt{e_{1}},-\alpha \sqrt{e_{2}}\right)$ with probabilities $\bar{p}_{i}^{*}$ where $\left(\bar{t}_{1 i}^{*}, \bar{t}_{2 i}^{*}\right)$ are the mass points in which the capacity achieving distribution for (31) is located with probabilities $\bar{p}_{i}^{*}$.

When the transmitter has unlimited battery, the energy that is allocated for message transmission can be saved in the battery and using the best-effort-transmit scheme in [4], [5], the following maximum rate is achievable:

$$
\begin{aligned}
\max & I\left(T_{1}, T_{2} ; Y\right) \\
\text { s.t. } & \mathbb{E}\left[p_{e_{1}} T_{1}^{2}+p_{e_{2}} T_{2}^{2}\right] \leq(1-\alpha) \mathbb{E}[E]
\end{aligned}
$$

where $T_{1}, T_{2}$ and $Y$ are related by the extended input channel relation in (31). The capacity achieving distribution for the problem in (32) is not easily obtained. Therefore, we resort 
to $T_{1}=T_{2}$ with a Gaussian distribution of zero mean and variance $(1-\alpha) \mathbb{E}[E]$. The resulting $(R, \Delta)$ pair is

$$
(R, \Delta)=(I(X ; X+\alpha E+N), I(\alpha E ; X+\alpha E+N))
$$

where $X \sim \mathcal{N}(0,(1-\alpha) \mathbb{E}[E])$.

\section{A Numerical Example}

In this section, we provide a numerical example of the optimal trade-off region $\mathcal{R}$ as well as the proposed suboptimal uncoded state amplification scheme under a binary energy arrival process with no battery and unlimited battery. In particular, $e_{1}=1, e_{2}=2.25$ with $p_{e_{1}}=0.8$, so that the energy arrival has entropy $H(E)=0.7219$ bits. The channel capacity with no battery and with unlimited battery are calculated as $C_{0}=0.5369$ bits and $C_{\infty}=\frac{1}{2} \log _{2}(1+\mathbb{E}[E])=0.5850$ bits, respectively. We observe that in the no battery case, the symmetric binary distribution of $\left(T_{1}, T_{2}\right)$ located at $\left(\sqrt{E_{1}}, \sqrt{E_{2}}\right)$ and $\left(-\sqrt{E_{1}},-\sqrt{E_{2}}\right)$ maximizes $I\left(T_{1}, T_{2} ; Y\right)$ and $h(Y)$ simultaneously. Therefore, the trade-off region generated by this symmetric binary distribution is the optimal trade-off region. We calculate the maximum entropy reduction in this case as $\Delta^{*}=0.5652$ bits. In the unlimited battery case, the boundary of the optimal $(R, \Delta)$ region is the line $R+\Delta=0.5850$ and in particular, $\Delta^{*}=0.5850$ bits as $H(E)>0.5850$. Note that $\Delta^{*}$ is higher in the unlimited battery case though battery blurs the energy arrival information. This is due to the fact that higher rates can be achieved with an unlimited battery. Moreover, note that lossless recovery of the state sequence at the receiver is not possible for no battery and unlimited battery cases since $\Delta^{*}$ is less than $H(E)$ in both cases. We plot the resulting trade-off regions and the points achievable by the proposed uncoded state amplification scheme in Fig. 2. Note that in the case of no battery if $I\left(T_{1}, T_{2} ; Y\right)$ and $h(Y)$ are maximized at different discrete distributions of $\left(T_{1}, T_{2}\right)$, then the optimal $(R, \Delta)$ region is a union of many regions.

We calculate the entropy reduction in the uncoded transmission case as $\Delta_{u c}=0.4466$ bits. As the energy splitting variable $\alpha$ is varied, we observe that the achieved $(R, \Delta)$ points travel from one edge to the other strictly interior to the optimal regions under no battery and unlimited battery cases. Therefore, in this case, digitizing the state sequence by means of channel codewords is optimal and analog state amplification performs suboptimally. Moreover, we observe that when there is no battery at the transmitter, even if the message transmission is performed at the capacity, there is a non-zero energy arrival information leakage to the receiver. In contrast, the receiver gets no information about the energy arrival process if transmitter has an unlimited battery and message transmission is performed at the capacity.

\section{CONCLUSION}

In this paper, we characterized the trade-off region between entropy reduction $\Delta$ of the energy arrivals and the message transmission rate $R$ in a communication system with an energy harvesting transmitter with no or unlimited battery. Shannon strategies achieve the boundary of the region in the no battery

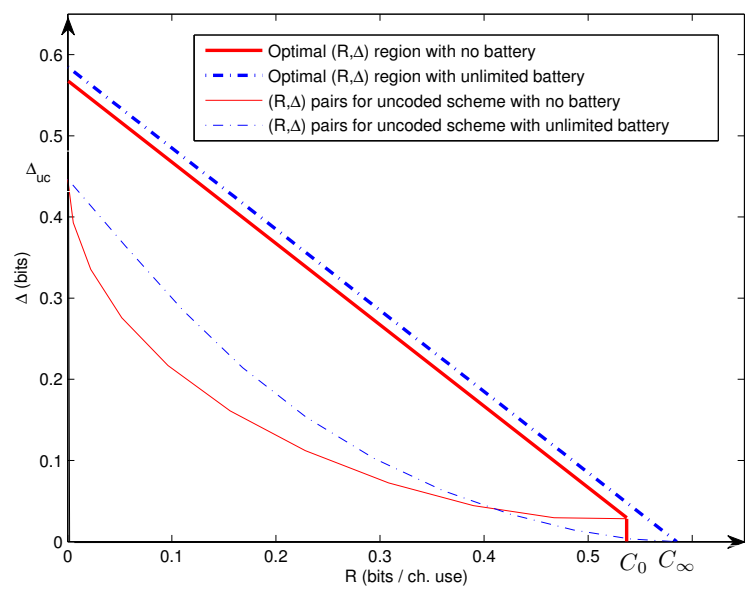

Fig. 2. $(R, \Delta)$ regions with optimal and suboptimal schemes.

case and we proved that the optimal input distributions are discrete. In the unlimited battery case, we showed that the optimal trade-off region can be expressed explicitly in a simple form and its boundary is achieved by a combination of besteffort-transmit and random binning schemes. We proposed an uncoded state amplification scheme and showed via a numerical example that digitizing the energy state performs significantly better than the uncoded scheme.

\section{REFERENCES}

[1] O. Ozel and S. Ulukus, "AWGN channel under time-varying amplitude constraints with causal information at the transmitter," in Asilomar Conference on Signals, Systems and Computers, November 2011.

[2] O. Ozel and S. Ulukus, "Capacity of the AWGN channel with a batteryless energy harvesting transmitter," IEEE Trans. on Information Theory, submitted, May 2012.

[3] J. G. Smith, "The information capacity of amplitude and varianceconstrained scalar Gaussian channels," Information and Control, vol. 18, pp. 203-219, April 1971.

[4] O. Ozel and S. Ulukus, "Information theoretic analysis of an energy harvesting communication system," in Workshop on Green Wireless (WGREEN) at IEEE PIMRC, September 2010.

[5] O. Ozel and S. Ulukus, "Achieving AWGN capacity under stochastic energy harvesting," IEEE Trans. on Information Theory, submitted, December 2010.

[6] A. Sutivong, M. Chiang, T. M. Cover, and Y.-H. Kim, "Channel capacity and state estimation for stte-dependent gaussian channels," IEEE Trans. on Inform. Theory, vol. 51, pp. 1486-1495, April 2005.

[7] Y.-H. Kim, A. Sutivong, and T. M. Cover, "State amplification," IEEE Trans. on Inform. Theory, vol. 54, pp. 1850-1859, April 2008.

[8] W. Zhang, S. Vedantam, and U. Mitra, "A constrained channel coding approach to joint communication and channel estimation," in IEEE ISIT, July 2008.

[9] C. Choudhuri, Y.-H. Kim, and U. Mitra, "Capacity-distortion trade-off in channels with state," in Allerton Conference, September 2010.

[10] C. Choudhuri, Y.-H. Kim, and U. Mitra, "Causal state amplification," in IEEE ISIT, August 2011.

[11] C. Shannon, "Channels with side information at the transmitter," IBM Jour. of Research and Development, vol. 2, October 1958.

[12] I. Abu-Faycal, M. Trott, and S. Shamai, "The capacity of discretetime memoryless rayleigh fading channels," IEEE Trans. on Information Theory, vol. 47, pp. 1290-1301, May 2001.

[13] T. H. Chan, S. Hranilovic, and F. Kschischang, "Capacity-achieving probability measure for conditionally Gaussian channels with bounded inputs," IEEE Trans. Inform. Theory, vol. 51, pp. 2073-2088, June 2005.

[14] N. Mehrav and S. Shamai, "Information rates subjected to state masking," IEEE Trans. on Information Theory, vol. 54, pp. 2254-2261, June 2007. 